\section{Riscos ocupacionais para o câncer de laringe: um estudo caso-controle}

\author{
Occupational risks for laryngeal cancer: \\ a case-control study
}

\author{
1 Faculdade de Medicina, \\ Universidade de São Paulo, \\ São Paulo, Brasil. \\ 2 Unit of Environmental \\ Cancer Epidemiology, \\ International Agency for \\ Research on Cancer, \\ Lyon, France. \\ 3 Faculdade de Saúde \\ Pública, Universidade de \\ São Paulo, São Paulo, Brasil. \\ ${ }^{4}$ Fundação Jorge Duprat \\ Figueiredo de Segurança e \\ Medicina do Trabalho, \\ São Paulo, Brasil. \\ 5 Centro de Tratamento e \\ Pesquisa, Hospital do Câncer \\ A. C. Camargo, São Paulo, \\ Brasil. \\ Correspondência \\ S. G. Sartor \\ Departamento de Medicina \\ Preventiva, Faculdade de \\ Medicina, Universidade de \\ São Paulo. \\ Av. Dr. Arnaldo 455, 2 o andar, \\ São Paulo, SP \\ 01246-903, Brasil. \\ sgs@usp.br
}

\section{Abstract}

The most solidly established risk factors for laryngeal cancer are tobacco and alcohol. As for occupational factors, the only established carcinogen is exposure to strong inorganic acid mists. However, asbestos, pesticides, paints, gasoline, diesel engine emissions, dusts, and other factors have been reported in the literature as occupational agents that increase the risk of laryngeal cancer. A hospital-based case-control study was conducted to investigate occupational risk factors for laryngeal cancer. Detailed data on smoking, alcohol consumption, and occupational history were collected for 122 laryngeal cancers and 187 controls matched by frequency (according to sex and age). Laryngeal cancer was associated with exposure to respirable free crystalline silica (OR = 1.83; 95\%CI: 1.00-3.36), soot (from coal, coke, fuel oil, or wood) (odds ratio - OR $=1.78 ; 95 \%$ confidence interval $-95 \% C I$ : 1.03-3.03), fumes (OR = 2.55; 95\%CI: 1.14-5.67), and live animals $(O R=1.80 ; 95 \%$ CI: 1.02-3.19).

Laryngeal Neoplasms; Risk Factors; Occupational Risks

\author{
Sergio Guerra Sartor 1 \\ José Eluf-Neto 1 \\ Noemie Travier ${ }^{2}$ \\ Victor Wünsch Filho 3 \\ Arline Sydneia Abel Arcuri 4 \\ Luís Paulo Kowalski 5 \\ Paolo Boffetta 2
}

\section{Introdução}

O câncer de laringe responde por 2,8\% dos novos casos de câncer em homens no mundo, e constitui a décima primeira neoplasia maligna mais freqüente, tendo uma estimativa de incidência ajustada por idade pela população mundial de 5,7/100 mil 1. Entre mulheres ele é menos freqüente, com uma estimativa de incidência ajustada por idade pela população mundial de 0,7/100 mil.

No volume mais recente (oitavo) do livro Cancer Incidence in Five Continents, o maior coeficiente de incidência, no sexo masculino, foi observado em Saragoça, Espanha (coeficiente ajustado por idade pela população mundial de 18,0/100 mil por ano) 2. Nesta publicação aparecem dois registros populacionais de câncer do Brasil: Campinas, São Paulo (7,4/100 mil por ano) e Goiânia, Goiás (6,4/100 mil por ano; coeficiente ajustado por idade pela população mundial, sexo masculino).

O câncer de laringe é responsável por aproximadamente 73.500 mortes por ano no mundo ${ }^{3}$. $\mathrm{Na}$ América do Sul tropical (inclui todos os países do continente, com exceção do Chile, Argentina e Uruguai), em 1990, o coeficiente de mortalidade por câncer de laringe padronizado por idade foi estimado em 3,9 para homens e 0,7 para mulheres (por 100 mil). Em levantamento realizado no Estado de São Paulo, em 1993, o câncer de laringe aparece como a oitava neoplasia mais freqüen- 
te entre as mortes por neoplasias malignas, com uma taxa bruta de 4,5/100 mil homens ${ }^{4}$.

O tabagismo e a ingestão de álcool são os fatores de risco mais bem estabelecidos para o câncer de laringe 5 . Ambos os fatores mostram uma forte relação dose-resposta com o risco de câncer de laringe, e um efeito sinérgico 5,6,7.

Entre os agentes ocupacionais, o único carcinógeno estabelecido para câncer de laringe é a exposição a névoas de ácidos inorgânicos fortes ${ }^{8}$. Tintas e gases de combustão de gasolina e diesel aparecem como agentes ocupacionais que aumentam o risco para o câncer de laringe 9 .

A poeira, como um agente químico ocupacional inespecífico, foi associada a um maior risco (odds ratio - OR = 1,6; intervalo de 95\% de confiança - IC95\%: 1,2-2,0) para o câncer de laringe em um estudo caso-controle realizado na Dinamarca, no qual foi feito ajuste para idade, tabagismo e etilismo ${ }^{10}$. Nesse mesmo estudo, ao se especificar o tipo de poeira, os riscos, embora elevados, não foram estatisticamente significantes. Para poeira de madeira, o risco de câncer de laringe associado também foi aumentado em outro estudo, embora sem significância estatística 11. Em Xangai, China, foi conduzido um estudo caso-controle de câncer de laringe de base populacional, no qual foram entrevistados 201 casos e 414 controles 12 . Foi observado um risco elevado (OR = 2,6; IC95\%: 1,4-4,8), ajustado por idade e tabagismo, para exposição à poeira de carvão.

A exposição ao óleo de corte foi avaliada em um estudo caso-controle, aninhado em uma coorte de trabalhadores de uma indústria automobilística, em Michigan, nos Estados Unidos, no período de 1917 a 1981 13. Nesse estudo foi observada uma relação dose-resposta para o óleo de corte, com o OR (ajustado por sexo, raça, ano de nascimento e idade) aumentando de 0,9 (IC95\%: 0,5-1,7) para indivíduos expostos a uma concentração de até $0,1 \mathrm{mg} / \mathrm{m}^{3}$ de ar, para 1,5 (IC95\%: 0,9-2,8) quando a exposição ocorria a uma concentração maior que 0,1 e até $0,5 \mathrm{mg} / \mathrm{m}^{3}$, e para 2,2 (IC95\%: 1,2-4,0) quando a exposição ocorria a uma concentração superior a $0,5 \mathrm{mg} / \mathrm{m}^{3}$.

Foram objetivos deste estudo investigar e quantificar o papel da exposição a determinados agentes ocupacionais no desenvolvimento do câncer de laringe e quantificar os riscos de câncer de laringe associados ao consumo de bebidas alcoólicas e ao hábito de fumar.

Este estudo integra um projeto multicêntrico, que abrange países do MERCOSUL (Argentina e Brasil) e da América Central (Cuba), denominado Estudo Internacional de Fatores Ambientais, Vírus e Câncer de Cavidade Oral e Laringe, coordenado pela International Agency for Research on Câncer (IARC)/Organização Mundial da Saúde, e que tem por objetivo investigar o papel de diversos fatores para o desenvolvimento de câncer de boca, faringe e laringe.

\section{Material e métodos}

Trata-se de um estudo caso-controle de base hospitalar. Foram considerados elegíveis todos os pacientes com diagnóstico de câncer de laringe, admitidos em quatro hospitais gerais e dois hospitais especializados em câncer, do Município de São Paulo, no período de fevereiro de 1999 a março de 2002. Os controles foram selecionados entre pacientes dos mesmos hospitais gerais em que os casos foram recrutados, com outras doenças que não câncer de laringe. Casos e controles deveriam residir na Região Metropolitana de São Paulo por um período superior a seis meses, para evitar viés de seleção.

Foram incluídos no estudo indivíduos com diagnóstico recente (que não tivessem iniciado qualquer tipo de tratamento específico) de câncer de laringe, confirmado por exame histopatológico. Os casos poderiam ter sido diagnosticados pela primeira vez fora dos hospitais participantes do estudo, desde que fossem encaminhados ao hospital participante sem tratamento para a doença. Casos de câncer de laringe consistiram de casos de câncer com o código C32, de acordo com a 10a revisão da Classificação Internacional de Doenças (CID-10) 14, incluindo todas as subcategorias: C32.0 - glote, C32.1 - região supraglótica, C32.2 - região subglótica, C32.3 - cartilagens da laringe, C32.8 - lesão invasiva da laringe e C32.9 - laringe sem outra especificação.

A identificação dos casos foi realizada através de pesquisa ativa, por meio de visitas periódicas aos departamentos/serviços onde os casos eram diagnosticados e tratados (cirurgia de cabeça e pescoço, otorrinolaringologia, laringoscopia etc.).

Os controles foram recrutados entre pacientes internados ou em atendimento ambulatorial nos mesmos hospitais dos casos, sem história ou suspeita de câncer de laringe. Os controles foram pareados segundo sexo e faixa etária, pela distribuição observada dos casos (emparelhamento por freqüência). Pacientes admitidos por doenças associadas positiva ou negativamente com fatores de risco conhecidos ou suspeitos para câncer de laringe não eram elegíveis.

Casos e controles foram entrevistados pessoalmente nos hospitais participantes do estudo, utilizando-se questionários padronizados que incluíam informações sócio-demográficas, história ocupacional detalhada, condições de moradia, tabagismo, consumo de álcool e mate, his- 
tória familiar de câncer, antecedente de doenças infecciosas selecionadas e padrões de dieta.

Além do questionário sobre hábitos de vida, foram utilizados dois tipos de questionário ocupacional. O primeiro compreendia uma descrição geral de todos os trabalhos do indivíduo. O segundo era constituído por um conjunto de questionários especializados voltados para ocupações (exemplo: ferramenteiro) ou atividades (exemplo: indústria química) que são de especial interessenesse estudo. Os(as) entrevistadores(as) foram treinados(as) para obter o máximo de informação que o indivíduo pudesse fornecer. Isso incluía a atividade da empresa, as matérias-primas, o produto final, as responsabilidades dos operários com a manutenção das máquinas, o tipo de construção em que eles trabalharam, as atividades dos colegas a sua volta, a presença de gases, fumos ou poeira, e outras informações que pudessem fornecer um indício de possível exposição a agentes químicos ou físicos.

O estudo foi apresentado aos indivíduos elegíveis como um inquérito de saúde não relacionado particularmente a câncer. As informações sobre o diagnóstico e o estadiamento clínico foram obtidas para cada caso de forma padronizada. Uma cópia do exame histológico era anexada ao questionário.

Uma equipe de profissionais com nível universitário e experiência em exposição ocupacional analisou as informações obtidas a partir dos questionários; para cada descrição de trabalho na história ocupacional, esses profissionais indicaram cada uma das substâncias presentes no ambiente de trabalho do indivíduo. Foi considerada exposição ocupacional quando a substância era encontrada num nível maior no ambiente de trabalho do que no meio ambiente. Para cada substância considerada presente, foram avaliados os seguintes quesitos: confiança (1. possível, 2. provável ou 3. certa), freqüência (1. de 1 a $5 \%, 2$. acima de 5 até $30 \%$ ou 3. acima de $30 \%$ da jornada de trabalho) e intensidade (1. inferior a $50 \%, 2$. de 50 a $150 \%$ ou 3 . acima de $150 \%$ do limite de tolerância de cada substância). A exposição a substâncias presentes no ambiente de trabalho foi avaliada sem conhecimento do status (caso ou controle) do indivíduo pesquisado.

O tamanho da amostra foi definido considerando-se que um $\alpha=0,05$ e uma prevalência de exposição de $20 \%$ entre os controles e a inclusão de 120 casos e 180 controles permitiria detectar um OR de câncer de laringe de 2,0 com poder estatístico de $74 \%$. Para uma prevalência de exposição de $10 \%$ entre os controles (também com $\alpha=0,05)$, esse tamanho de amostra teria um poder estatístico de $80 \%$ para detectar $\mathrm{OR}=2,5$.
Para construir uma variável que contemplasse o total de tabaco consumido por cada indivíduo, foi considerado que cada cigarro contém $1 \mathrm{~g}$ de tabaco; charuto, $4 \mathrm{~g}$; e cachimbo, $3 \mathrm{~g}{ }^{15}$. Calculou-se, então, o consumo médio diário de tabaco em gramas, que foi dividido por 20 (quantidade de tabaco, em gramas, de um maço de cigarros), e multiplicado pelo número de anos de tabagismo de cada indivíduo, encontrando-se assim o valor de maços-ano.

De modo análogo, para dose acumulada de álcool foi considerado que cerveja contém $5 \%$ de álcool; vinho, 12\%; aguardente, uísque, vodka e rum, $41 \%$ e; licor, $30 \%$. As quantidades, em litros de álcool, encontradas a partir dessas porcentagens, foram transformadas em gramas de álcool considerando-se que cada litro de álcool contém 798g. Foi então calculado o consumo médio diário em gramas de álcool que foi multiplicado pelo número de anos de consumo chegando-se assim à variável expressa em gramas-ano.

Tanto para consumo de álcool como para tabagismo, a variável que representa a dose acumulada foi dividida em quatro categorias, segundo os pontos de corte referentes aos quartis da variável, observados entre os controles.

Com relação às variáveis ocupacionais, foram considerados efetivamente expostos os indivíduos que receberam pontuação 2 ou 3, no quesito confiança, para uma determinada exposição ocupacional; os indivíduos com pontuação 1, nesse quesito, foram considerados não expostos. Para calcular a exposição acumulada de cada agente ocupacional específico, multiplicou-se o período (em anos) de exposição por oito horas (jornada diária), por cinco dias (uma semana de trabalho), por cinqüenta semanas (um ano), pela freqüência e pela intensidade.

Essa variável contínua (exposição acumulada), de cada agente ocupacional específico, foi dividida em três categorias (baixa, média e alta) segundo pontos de corte referentes aos tercis da variável, considerando a exposição entre os 597 indivíduos (casos de câncer oral, câncer de laringe e controles), inclusos no projeto da IARC no centro de São Paulo, e para os quais havia codificação ocupacional disponível. A inclusão também da exposição observada entre os casos de câncer deveu-se ao número muito reduzido de indivíduos expostos a alguns agentes ocupacionais.

Para estimar o risco de câncer de laringe associado com as variáveis de interesse, foram calculados os OR e IC95\% por regressão logística não condicional 16, com o programa Stata versão 8.0 (Stata Corporation, College Station, Estados Unidos) . 
A significância estatística foi avaliada por meio do teste da razão de verossimilhança. Consideraram-se como nível de significância para rejeitar a hipótese nula valores menores que 0,05. Para variáveis categóricas ordenáveis, foram realizados testes de tendência linear nos OR, considerando os escores das categorias como contínuos 16.

Variáveis de confusão também foram avaliadas por regressão logística não condicional. Como casos e controles foram emparelhados por sexo e faixa etária (pareamento por freqüência), os OR foram sempre ajustados por essas duas variáveis. Sexo entrou no modelo de forma dicotômica e idade entrou em quatro categorias (35-49, 50-59, 60-69 e 70-79 anos).

O projeto do estudo foi aprovado pela Comissão Nacional de Ética em Pesquisa (CONEP) e pelos comitês de ética de todos os hospitais participantes. Os indivíduos foram incluídos no estudo ao assinarem o Termo de Consentimento Pós-informação.

\section{Resultados}

Dos 202 casos e 242 controles potencialmente elegíveis, identificados nos hospitais participantes, não foram incluídos 80 casos e 55 controles. As causas de não inclusão foram: local de residência (41 casos e 20 controles), diagnóstico não confirmado (23 casos e 11 controles), recusa (5 casos e 20 controles), tratamento anterior (6 casos e 1 controle), condição física (5 casos e 1 controle) e indisponibilidade do paciente ( 2 controles).

Dessa forma, foram incluídos 122 casos e 187 controles. Com relação ao diagnóstico, os controles se distribuíam por diversas categorias da CID-10 14. Observou-se uma maior proporção de controles com doenças do aparelho circulatório (28\%), seguidas pelas doenças do aparelho digestivo (16\%) e geniturinário (13\%), que são os grupos de doenças que levam a uma maior demanda por atendimento hospitalar. Pacientes com neoplasias constituíram menos de $10 \%$ dos controles.

Dentre os casos, 104 eram do sexo masculino e 18 do sexo feminino, e dentre os controles 142 e 45 , respectivamente. A idade dos casos variou de 39 a 79 anos, com média igual a 60 anos (desvio padrão $=10$ anos), enquanto que entre os controles a idade variou de 38 a 79 anos, com média igual a 58 anos (desvio padrão = 11 anos).

Proporções semelhantes de casos (18\%) e de controles (17\%) não freqüentaram escola. Contudo, maior escolaridade (ensino médio/ensino superior) esteve associada, sem significância estatística, ao menor risco de câncer de laringe (OR = 0,39; IC95\%: 0,14-1,12).

A maioria dos participantes relatou consumir ou ter consumido álcool, pelo menos uma vez por mês, $87 \%$ dos casos e $71 \%$ dos controles (Tabela 1). Essa diferença de consumo entre os dois grupos representa um risco superior a dois para os indivíduos que consomem ou consumiram álcool. O risco de câncer de laringe aumenta com a quantidade de álcool consumido ( $\mathrm{p}$ de tendência linear igual a 0,002).

Indivíduos que fumam ou fumaram, em média, um cigarro, charuto ou cachimbo diariamente, por pelo menos um ano, foram considerados tabagistas. Apenas $6 \%$ dos casos e $27 \%$ dos controles eram não tabagistas (Tabela 1). Os tabagistas têm risco quadruplicado, quando comparados aos não-tabagistas. Observa-se um risco crescente de câncer de laringe segundo o número de maços-ano.

As exposições ocupacionais a hidrocarbonetos aromáticos policíclicos, poeira de madeira, névoa de óleo mineral, aguarrás mineral e aldeídos foram as mais freqüentes. Os agentes foram classificados em seis grupos: poeiras, fumos, vapores, névoas, biológicos e outros.

A Tabela 2 mostra o número de indivíduos expostos e os respectivos OR de câncer de laringe para os agentes ocupacionais que se mostraram associados à doença de maneira estatisticamente significante. Para os demais agentes pesquisados (poeiras em geral, poeira de isolamento inorgânico, fibras minerais manufaturadas, asbesto, poeira abrasiva, areia, poeira de concreto, poeira de tijolo, poeira de carvão mineral, poeira de coque, poeira de carvão vegetal, poeira de negro de fumo, poeira de madeira, poeira de cromato, poeira de cromo e seus compostos, poeira de níquel e seus compostos, poeira de aço doce, poeira de liga dura, pigmentos inorgânicos, poeira de tecidos naturais, poeira de tecidos sintéticos, fumos de cromato, fumos de cromo e seus compostos, fumos de níquel e seus compostos, fumos de combustão, emissão de motores, fumos de asfalto e betume, fumos de alcatrão e piche, fumos de creosoto, fumos de plásticos e borrachas, hidrocarbonetos aromáticos policíclicos, fumos de soldagem, petróleo e gasolina, querosene e óleo diesel, aguarrás mineral, álcool, aldeídos, solventes orgânicos clorados, névoas em geral, arsênico e compostos, névoa de ácido inorgânico forte, névoa de óleo mineral, pesticidas, carnes, radiações ionizantes e voz profissional) não se observou associação entre a exposição e o risco de câncer de laringe.

Entre as poeiras, a exposição à fuligem (Tabela 2) esteve associada de forma estatisticamente significante ao câncer de laringe. Outra associa- 
Odds ratios (OR) ajustados de câncer de laringe segundo consumo de álcool e tabagismo.

\begin{tabular}{lccc}
\hline Consumo de álcool e tabagismo & Casos & Controles & OR (IC95\%) \\
\hline Consumo de álcool (gramas/ano) & 16 & 52 & \\
$\quad$ Nunca & 106 & 126 & Grupo de referência \\
Sim & 10 & 31 & $2,28(1,09-4,79)$ \\
Até 270 & 23 & 30 & $1,24(0,46-3,38)$ \\
$>$ 270-890 & 24 & 32 & $2,09(0,84-5,21)$ \\
$>890-1.940$ & 46 & 30 & $1,81(0,73-4,50)$ \\
$>1.940$ & & & $3,68(1,55-8,71)$ \\
Tendência linear & & & $z=3,08 ; p=0,002$ \\
Tabagismo (maços/ano) & 7 & 50 & \\
$\quad$ Nunca & 112 & 133 & Grupo de referência \\
Sim & 16 & 32 & $4,20(1,73-10,22)$ \\
Até 18,0 & 23 & 30 & $2,78(0,97-7,94)$ \\
$>18,0-29,5$ & 23 & 31 & $4,52(1,62-12,60)$ \\
$>$ 29,5-43,5 & 50 & 32 & $3,68(1,31-10,36)$ \\
$>43,5$ & & & $7,52(2,82-19,99)$ \\
Tendência linear & & $z=4,04 ; p<0,001$
\end{tabular}

Nota: três casos e quatro controles sem informação quantitativa para consumo de álcool e nove controles sem informação quantitativa para tabagismo.

OR de consumo de álcool ajustados por sexo, idade e tabagismo; OR de tabagismo ajustados por sexo, idade e consumo de álcool.

ção que merece destaque neste grupo é com a exposição à sílica cristalina livre respirável que mostra um risco quase que dobrado para os expostos quando comparados aos não expostos.

A exposição a fumos em geral eleva em aproximadamente duas vezes e meia o risco de câncer de laringe, com significância estatística (OR = 2,55; IC95\%: 1,14-5,67). Contudo, observando-se a exposição a fumos específicos, nenhum se associou de forma estatisticamente significante ao risco de câncer de laringe (dados não mostrados).

A exposição a carnes eleva o risco em uma vez e meia, com efeito dose-resposta, chegando a quase três vezes ( $\mathrm{OR}=2,80$; IC95\%: 0,96-8,16) para o grupo com alta exposição (dados não mostrados). A exposição a animais vivos mostrou-se associada ao câncer de laringe, de maneira estatisticamente significante, tanto na forma dicotômica como no grupo considerado com alta exposição, apresentando também efeito dose-resposta (Tabela 2). Com relação às subcategorias desse agente, o risco (ajustado por sexo, idade, tabagismo e consumo de álcool) é aproximadamente dois, e é estatisticamente significante para as três subcategorias específicas: boi $(\mathrm{OR}=2,00$; IC95\%: 1,01-3,95); frango (OR = 1,99; IC95\%: 1,09-3,66) e porco $(\mathrm{OR}=1,87$; IC95\%: 1,01-3,47).
Apenas dois casos apresentaram exposição a radiações ionizantes e um caso relatou uso profissional da voz.

\section{Discussão}

O fator de risco mais importante para câncer de laringe é o tabagismo, e essa relação é conhecida desde a década de 50, quando foram publicados os primeiros estudos epidemiológicos sobre essa neoplasia 5 . Vários estudos encontraram riscos aumentados para câncer de laringe associado ao tabagismo 9,11,12,17,18,19,20,21. No presente estudo, tabagismo esteve fortemente associado ao risco de câncer de laringe (OR = 4,2; IC95\%: 1,7-10,2; ajustado por sexo, idade e consumo de álcool). Além disso, observou-se também relação doseresposta: indivíduos que fumaram mais do que 43,5 maços-ano tiveram um risco 7,5 vezes maior de câncer de laringe, quando comparados com não fumantes.

Neste estudo, o consumo de álcool esteve significativamente associado ao câncer de laringe (OR = 2,3; IC95\%: 1,1-4,8; ajustado por sexo, idade e tabagismo), em conformidade com o observado em diversas investigações 10,12,17,18,19,20,21,22,23. Indivíduos que consumiram mais do que 1.940 
Odds ratio (OR) de câncer de laringe segundo agentes ocupacionais selecionados.

\begin{tabular}{|c|c|c|c|c|}
\hline & Casos & Controles & OR (IC95\%) * & OR (IC95\%) ** \\
\hline \multicolumn{5}{|c|}{ Sílica cristalina livre respirável } \\
\hline Não & 81 & 151 & Grupo de referência & \\
\hline Sim & 41 & 36 & $1,90(1,10-3,28)$ & $1,83(1,00-3,36)$ \\
\hline Baixo & 15 & 14 & $1,91(0,86-4,24)$ & $1,80(0,75-4,34)$ \\
\hline Médio & 11 & 14 & $1,31(0,56-3,09)$ & $1,36(0,53-3,49)$ \\
\hline Alto & 15 & 8 & $1,86(1,14-7,18)$ & $2,67(0,95-7,50)$ \\
\hline Tendência linear & & & & $z=1,97 ; p<0,05$ \\
\hline \multicolumn{5}{|c|}{$\begin{array}{l}\text { Fuligem (de carvão mineral, coque, } \\
\text { madeira, óleo combustível) }\end{array}$} \\
\hline Não & 60 & 110 & Grupo de referência & \\
\hline Sim & 62 & 77 & $1,28(0,80-2,06)$ & $1,78(1,03-3,03)$ \\
\hline Baixo & 18 & 32 & $1,00(0,51-1,94)$ & $1,55(0,73-3,30)$ \\
\hline Médio & 22 & 25 & $1,29(0,65-2,55)$ & $1,62(0,75-3,50)$ \\
\hline Alto & 22 & 19 & $1,81(0,89-3,68)$ & $2,42(1,08-5,46)$ \\
\hline Tendência linear & & & & $z=2,27 ; p=0,02$ \\
\hline \multicolumn{5}{|l|}{ Animais vivos } \\
\hline Não & 78 & 133 & Grupo de referência & \\
\hline Sim & 44 & 54 & $1,26(0,77-2,07)$ & $1,80(1,02-3,19)$ \\
\hline Baixo & 14 & 19 & $1,04(0,49-2,23)$ & $1,50(0,64-3,52)$ \\
\hline Médio & 15 & 20 & $1,19(0,56-2,50)$ & $1,55(0,67-3,58)$ \\
\hline Alto & 15 & 15 & $1,66(0,76-3,63)$ & $2,64(1,08-6,44)$ \\
\hline Tendência linear & & & & $z=2,24 ; p=0,02$ \\
\hline
\end{tabular}

Nota: um controle sem informação quantitativa para o agente fuligem.

* Ajustado por sexo e idade;

** Ajustado por sexo, idade, tabagismo e consumo de álcool. Neste modelo foram excluídos os 15 indivíduos sem informação quantitativa sobre tabagismo e/ou consumo de álcool.

gramas/ano de álcool tiveram um risco 3,7 vezes maior de câncer de laringe, quando comparados com indivíduos que não consumiam álcool.

Quatro exposições ocupacionais se mostraram significativamente associadas ao risco de câncer de laringe: sílica cristalina livre respirável, fuligem (de carvão mineral, coque, madeira, óleo combustível), fumos em geral e animais vivos.

A associação encontrada no presente estudo entre sílica cristalina livre respirável e câncer de laringe é confirmada pelo estudo de Imbernon et al. 24, no qual foi relatado um OR de 2,0 (IC95\%: 1,1-3,5). Entretanto, outros estudos não encontraram associação ao pesquisar sílica ou quartzo (um tipo de sílica cristalina) como fator de risco ocupacional para câncer de laringe 25,26,27 .

Não é do nosso conhecimento outro estudo epidemiológico que tenha investigado especificamente exposição à fuligem como fator de risco para câncer de laringe. Entretanto, a fuligem faz parte da história da medicina do trabalho como carcinógeno ocupacional. Há mais de 200 anos, um cirurgião londrino, Percivall Pott, estabeleceu o nexo causal entre o câncer de escroto e o trabalho de limpeza de chaminés 28 . A IARC classifica a fuligem como agente ocupacional com suficiente evidência de carcinogenicidade para humanos a partir de diversos estudos que analisaram a associação desse agente com câncer de pele, pulmão, esôfago, fígado e leucemia 29.

Outra associação com exposição ocupacional encontrada neste estudo foi com fumos em geral (OR = 2,55; IC95\%: 1,14-5,67; ajustado por sexo, idade, consumo de álcool e tabagismo). Nos estudos de Bravo et al. 30 e de Ahrens et al. 19, que abordaram fumos como exposição ocupacional inespecífica, não se observou associação com o câncer de laringe. Vários estudos avaliaram o efeito da exposição a fumos específicos. Muscat \&Wynder 11 e Goldberg et al. 25 encontraram associação estatisticamente significante, entre fumos de diesel e câncer de laringe. Outros estu- 
dos encontraram associação, sem significância estatística, para fumos de fundição, gasolina e diesel ${ }^{9}$ e para fumos de soldagem 26 .

No presente estudo, indivíduos que relataram exposição a animais vivos apresentaram risco aumentado de câncer de laringe $(\mathrm{OR}=1,80$; IC95\%: 1,02-3,19; ajustado por sexo, idade, consumo de álcool e tabagismo), com efeito dose-resposta. A exposição a inseticidas utilizados na criação de animais (OR de câncer de laringe = 2,03; IC95\%: 0,87-4,75; dados não apresentados) poderia ser responsável por parte da associação entre câncer de laringe e exposição a animais vivos. Ao incluir no modelo multivariado o ajuste por exposição a inseticidas utilizados na criação de animais, observou-se pequena redução no risco associado à exposição a animais (OR = 1,63; IC95\%: 0,892,98). Outra possível explicação seria a exposição a microorganismos (especialmente os vírus) e endotoxinas presentes nos ambientes de criação de animais 31,32 .

Goldberg et al. 33 , averiguando se determinadas ocupações estavam associadas ao risco de cânceres de laringe e hipofaringe, encontraram $\mathrm{OR}=1,6(0,05<\mathrm{p}<0,10)$ desses dois cânceres no grupo de ocupações que abrigava agricultores e criadores de animais. Apesar de parte desse risco poder estar relacionada à exposição a animais vivos, é impossível distinguir até que ponto ela é responsável por esse aumento de risco, uma vez que as ocupações foram agrupadas.

$\mathrm{O}$ aumento de risco em indivíduos com exposição ocupacional a carnes, encontrado neste estudo, pode ser confrontado com resultados de duas investigações, embora elas tenham analisado exposições ocupacionais simplesmente como ocupações. Goldberg et al. 33 não encontraram associação entre a ocupação de açougueiro e câncer de laringe, enquanto no estudo de De Stefani et al. 34 o risco foi próximo a três $(\mathrm{OR}=2,8$; IC95\%: 1,1-7,2).

A exposição à névoa de ácido inorgânico forte não esteve associada ao risco de câncer de laringe. Apesar deste agente ocupacional ser classificado como carcinogênico para laringe pela IARC, outros autores também não encontraram associação entre essa exposição ocupacional e câncer de laringe 9,12,26. A falta de poder estatístico pode explicar a não associação do risco de câncer de laringe com a exposição à névoa de ácido inorgânico forte e a outros agentes existentes no ambiente de trabalho.

Por outro lado, deve ser considerada a questão de múltiplas comparações (no presente estudo, foram investigados 71 agentes ocupacionais específicos e 26 agentes ocupacionais não específicos), que aumentam a chance de encontrar associações estatisticamente significantes.

Em estudos caso-controle, a seleção dos participantes constitui uma grande preocupação. Para os casos selecionados nos hospitais especializados, foram recrutados controles em hospitais gerais que atendiam população proveniente da mesma área ou população similar.

$\mathrm{Na}$ avaliação da exposição ocupacional do presente estudo foi utilizada uma nova metodologia 35 . Com esta metodologia, em que as possíveis exposições no ambiente de trabalho são avaliadas de modo aprofundado, pretendeu-se reduzir o erro de classificação não diferencial da exposição. Por outro lado, o uso dessa metodologia depende da aplicação de diversos questionários, o que prolonga o tempo de entrevista. Neste estudo, a duração média das entrevistas foi de 85 minutos, sendo que $67 \%$ das entrevistas tiveram duração superior a uma hora. Essa longa duração pode ter cansado o entrevistado e levado a um prejuízo na qualidade da informação prestada.

$O$ viés de classificação diferencial da exposição, atribuível à atividade de codificação ocupacional, foi evitado pelo mascaramento dos codificadores quanto ao status do indivíduo, ou seja, o codificador não tinha conhecimento se se tratava de caso ou controle o indivíduo cuja entrevista estava sendo avaliada.

O presente estudo confirmou o tabagismo e o consumo de álcool como fatores de risco importantes para o desenvolvimento do câncer de laringe. As associações entre exposição ocupacional aos agentes sílica cristalina livre respirável, fuligem (de carvão mineral, coque, madeira, óleo combustível), fumos em geral e animais vivos, e câncer de laringe, não são explicadas por padrões de tabagismo ou consumo de álcool. 


\section{Resumo}

O tabagismo e o consumo de álcool são os fatores de risco mais bem estabelecidos para o câncer de laringe. Com relação aos fatores ocupacionais, o único carcinógeno estabelecido é a exposição a névoas de ácidos inorgânicos fortes. Entretanto, asbesto, pesticidas, tintas, gases de combustão de gasolina e diesel e poeiras, entre outros, aparecem na literatura como agentes ocupacionais que aumentam o risco de câncer de laringe. Um estudo caso-controle de base hospitalar foi conduzido para investigar fatores de risco ocupacionais para câncer de laringe. Foram coletadas informações detalhadas sobre tabagismo, consumo de álcool e história ocupacional de 122 casos de câncer de laringe e 187 controles pareados por freqüência (segundo sexo e idade). Encontrou-se risco aumentado de câncer de laringe nos indivíduos com exposição à sílica cristalina livre respirável (OR = 1,83; IC95\%: 1,00-3,36), à fuligem (de carvão mineral, coque, madeira, óleo combustivel) (OR = 1,78; IC95\%: 1,03-3,03), a fumos em geral $(O R=2,55$; IC95\%: 1,14-5,67) e a animais vivos (OR $=1,80 ;$ IC95\%: $1,02-3,19$ ).

Neoplasias Laríngeas; Fatores de Risco; Riscos Ocupacionais

\section{Colaboradores}

S. G. Sartor e J. Eluf-Neto colaboraram na coordenação do trabalho de coleta de dados, na análise estatística e na redação do artigo. P. Boffetta contribuiu no planejamento do estudo e na análise estatística. N. Travier trabalhou na checagem da consistência dos dados e na análise estatística. V. Wünsch Filho participou do planejamento do estudo e na coordenação do trabalho de coleta de dados. A. S. A. Arcuri colaborou na codificação da exposição ocupacional. L. P. Kowalski contribuiu na coleta de dados e na revisão do artigo.

\section{Agradecimentos}

Comunidade Econômica Européia (nº. IC18CT970222) e Fundação de Amparo à Pesquisa do Estado de São Paulo (no. 2001/01768-2).

\section{Referências}

1. Parkin DM, Pisani P, Ferlay J. Estimates of the worldwide incidence of 25 major cancers in 1990. Int J Cancer 1999; 80:827-41.

2. Parkin DM, Whelan SL, Ferlay J, Teppo L, Thomas DB, editors. Cancer incidence in five continents. v. 8. Lyon: International Agency for Research on Cancer; 2002. (Scientific Publication, 155).

3. Pisani P, Parkin DM, Bray F, Ferlay J. Estimates of the worldwide mortality from 25 cancers in 1990. Int J Cancer 1999; 83:18-29.

4. Fonseca LAM. Mortalidade por câncer no estado de São Paulo: perfil atual, distribuição geográfica e tendências temporais [Tese de Doutorado]. São Paulo: Faculdade de Saúde Pública, Universidade de São Paulo; 1996.

5. Austin DF, Reynolds P. Laryngeal cancer. In: Schottenfeld D, Fraumeni Jr. JF, editors. Cancer epidemiology and prevention. 2nd Ed. New York: Oxford University Press; 1996. p. 619-36.

6. Hill C. Épidémiologie des cancers des voies aérodigestives supérieures. Bull Cancer 2000; 5:5-8.
7. Rafferty MA, Fenton JE, Jones AS. The history, aetiology and epidemiology of laryngeal carcinoma. Clin Otolaryngol 2001; 26:442-6.

8. International Agency for Research on Cancer. Monographs on the evaluations of carcinogenic risks to humans: occupational exposures to mists and vapours from strong inorganic acids; and other industrial chemicals. v. 54. Lyon: International Agency for Research on Cancer; 1992.

9. Brown LM, Mason JT, Pickle WL, Stewart PA, Buffler PA, Burau K, et al. Occupational risk factors for laryngeal cancer on the Texas Gulf Coast. Cancer Res 1988; 48:1960-4.

10. Olsen J, Sabroe S. Occupational causes of laryngeal cancer. J Epidemiol Community Health 1984; 38:117-21.

11. Muscat JE, Wynder EL. Tobacco, alcohol, asbestos, and occupational risk factors for laryngeal cancer. Cancer 1992; 69:2244-51. 
12. Zheng W, Blot WJ, Shu X, Gao Y, Ji B, Ziegler RG, et al. Diet and other risk factors for laryngeal cancer in Shanghai, China. Am J Epidemiol 1992; 136:17891.

13. Eisen EA, Tolbert PE, Hallock MF, Monson RR, Smith TJ, Woskie SR. Mortality studies of machining fluid exposure in the automobile industry III: a case-control study of larynx cancer. Am J Ind Med 1994; 26:185-202.

14. Organização Mundial da Saúde. Classificação estatística internacional de doenças e problemas relacionados à saúde - 10a revisão. v. 1. São Paulo: Centro Colaborador da OMS para a Classificação de Doenças em Português; 1995.

15. Boffetta P, Pershagen G, Jöckel K, Forastiere F, Gaborieau V, Heinrich J, et al. Cigar and pipe smoking and lung cancer risk: a multicenter study from Europe. J Natl Cancer Inst 1999; 91:697-701.

16. Breslow NE, Day NE. Statistical methods in cancer research: the analysis of case-control studies. v. 1. Lyon: International Agency for Research on Cancer; 1980. (Scientific Publications, 32).

17. De Stefani E, Correa P, Oreggia F, Leiva J, Rivero S, Fenandez G, et al. Risk factors for laryngeal cancer. Cancer 1987; 60:3087-91.

18. Falk RT, Pickle LW, Brown LM, Mason TJ, Buffler PA, Fraumeni Jr. JF. Effect of smoking and alcohol consumptions on laryngeal cancer risk in coastal Texas. Cancer Res 1989; 49:4024-9.

19. Ahrens W, Jöckel K, Patzak W, Elsner G. Alcohol, smoking, and occupational factors in cancer of the larynx: a case-control study. Am J Ind Med 1991; 20:477-93.

20. Maier H, Tisch M. Epidemiology of laryngeal cancer: results of the Heidelberg case-control study. Acta Otolaryngol 1997; 527 Suppl:160-4.

21. Suarez-Varela MMM, Gonzalez AL, Guillem EM, Selva MIM, Miñana IV, Gisbert V. Hábitos tóxicos en relación con el cáncer de laringe. Acta Otorrinolaringol Esp 1997; 48:45-50.

22. Hedberg K, Vaughan TL, Whitte E, Davis S, Thomas DB. Alcoholism and cancer of the larynx: a casecontrol study in western Washington (United States). Cancer Causes Control 1994; 5:3-8.

23. Tavani A, Negri E, Franceschi S, Barbone F, La Vecchia C. Attributable risk for laryngeal cancer in northern Italy. Cancer Epidemiol Biomarkers Prev 1994; 3:121-5.

24. Imbernon E, Goldberg M, Bonenfant S, Chevalier A, Guenel P, Vatré R, et al. Occupational respiratory cancer and exposure to asbestos: a case control study in a cohort of workers in the electricity and gas industry. Am J Ind Med 1995; 28:339-52.
25. Goldberg M, Goldberg P, Leclerc A, Chastang J, Marne M, Dubordieu D. A 10-year incidence survey of respiratory cancer and a case-control study within a cohort of nickel mining and refining workers in New Caledonia. Cancer Causes Control 1994; 5:15-25.

26. Gustavsson P, Jakobsson R, Johansson H, Lewin F, Norell S, Rutkvist L. Occupational exposures and squamous cell carcinoma of the oral cavity, pharynx, larynx, and oesophagus: a case-control study in Sweden. Occup Environ Med 1998; 55:393-400.

27. Laforest L, Luce D, Goldberg P, Denis B, Gérin M, Demers PA, et al. Laryngeal and hypopharyngeal cancers and occupation exposure to formaldehyde and various dusts: a case-control study in France. Occup Environ Med 2000; 57:767-73.

28. Mendes R. Aspectos históricos da patologia do trabalho. In: Mendes R, organizador. Patologia do trabalho. Rio de Janeiro: Editora Atheneu; 1995. p. 3-31.

29. International Agency for Research on Cancer. Monographs on the evaluations of carcinogenic risks to humans. Overall evaluations of carcinogenicity: an updating of IARC monographs. Lyon: International Agency for Research on Cancer; 1987.

30. Bravo MO, Spinosa J, Calero JR. Occupational risk factors for cancer of the larynx in Spain. Neoplasma 1990; 37:477-81.

31. Thu K, Zwerling C, Donham K. Problemas de salud y pautas patológicas. In: Organización Internacional del Trabajo, editor. Enciclopedia de salud y seguridad en el trabajo. v. 3. Madrid: Ministerio de Trabajo y Asuntos Sociales; 1998. p. 70.7-11.

32. Franco Netto G. On the need to assess cancer risk in populations environmentally and occupationally exposed to virus and chemical agents in developing countries. Cad Saúde Pública 1998; 14 Suppl 3:87-98.

33. Goldberg P, Leclerc A, Luce D, Morcet JF, Brugere J. Laryngeal and hypopharyngeal cancer and occupation: results of a case-control study. Occup Environ Med 1997; 54:477-82.

34. De Stefani E, Boffetta P, Oreggia F, Ronco A, Kogevinas M, Mendilaharsu M. Occupation and the risk of laryngeal cancer in Uruguay. Am J Ind Med 1998; 33:537-42.

35. Siemiatycki J. Risk factors for cancer in the workplace. Boca Raton: CRC Press; 1991.

Recebido em 06/Abr/2006

Versão final reapresentada em 02/Jan/2007

Aprovado em 11/Jan/2007 